\title{
Acupuncture Improves Sleep Conditions of Minipigs Representing Diurnal Animals through an Anatomically Similar Point to the Acupoint (GV20) Effective for Humans
}

\author{
Ka-ichiro Takeishi, ${ }^{1}$ Masahisa Horiuchi, ${ }^{1}$ Hiroaki Kawaguchi, ${ }^{2}$ \\ Yoshiki Deguchi, ${ }^{3}$ Hiroyuki Izumi, ${ }^{3}$ Emi Arimura, ${ }^{1,4}$ Satoshi Kuchiiwa, ${ }^{5}$ \\ Akihide Tanimoto, ${ }^{6}$ and Toru Takeuchi ${ }^{1}$ \\ ${ }^{1}$ Department of Environmental Medicine, Graduate School of Medical and Dental Sciences, Kagoshima University, \\ 8-35-1 Sakuragaoka, Kagoshima 890-8544, Japan \\ ${ }^{2}$ Department of Veterinary Pathology, Faculty of Agriculture, Kagoshima University, 1-21-24 Korimoto, Kagoshima 890-0065, Japan \\ ${ }^{3}$ Drug Safety Research Laboratories, Shin Nippon Biomedical Laboratories, Ltd., 2438 Miyanoura, Kagoshima 891-1394, Japan \\ ${ }^{4}$ Department of Life and Environmental Science, Kagoshima Prefectural College, 1-52-1 Shimo-Ishiki, Kagoshima 890-0005, Japan \\ ${ }^{5}$ Department of Neuroanatomy, Graduate School of Medical and Dental Sciences, Kagoshima University, \\ 8-35-1 Sakuragaoka, Kagoshima 890-8544, Japan \\ ${ }^{6}$ Department of Molecular and Cellular Pathology, Graduate School of Medical and Dental Sciences, Kagoshima University, \\ 8-35-1 Sakuragaoka, Kagoshima 890-8544, Japan
}

Correspondence should be addressed to Masahisa Horiuchi, masakun@m.kufm.kagoshima-u.ac.jp

Received 27 September 2011; Revised 1 December 2011; Accepted 1 December 2011

Academic Editor: Ke Ren

Copyright ( $) 2012$ Ka-ichiro Takeishi et al. This is an open access article distributed under the Creative Commons Attribution License, which permits unrestricted use, distribution, and reproduction in any medium, provided the original work is properly cited.

\begin{abstract}
Acupuncture, an alternative medicine, has been widely applied for people with sleep disturbances; therefore, the effects should be evaluated objectively. Micro-minipigs (MMPigs), the smallest miniature pigs for animal experiments, were used. Acupuncture was performed at two different points: Dafengmen is located on the head and is an anatomically similar point to human-Baihui (GV20), an effective acupoint for sleep disturbances in humans; pig-Baihui is on the back. The procedure was performed as follows: shallow, within $5 \mathrm{~mm}$ depth for several seconds; deep, $10-20 \mathrm{~mm}$ depth for $20 \mathrm{~min}$. The sleep conditions were evaluated by actigraph, and the amount of catecholamine in pooled urine after acupuncture treatment. MMPigs with deep acupuncture at Dafengmen showed significantly efficient values on actigraph and catecholamine analysis as compared with untreated MMPigs. The effective acupoint for sleep conditions in the porcine model is at an anatomically similar point to humans, rather than the point determined by traditional Chinese medicine.
\end{abstract}

\section{Introduction}

People with sleep disturbances have increased in developed countries $[1,2]$. Not only aged people but also working people often show sleep disturbances, causing severe problems in industry $[3,4]$. Additionally, from the viewpoint of medical economics, the costs of treating sleep disturbances have stressed the government budget, so efficient treatment for sleep disturbance is required $[3,4]$.

Pharmacological drugs involved in serotonin and melatonin metabolism have been applied for people with sleep disturbances $[5,6]$; however, the number of people with sleep disturbances has not tended to decrease. At present, not only pharmacological drugs but also alternative medicines are administered [7]. Acupuncture, an alternative medicine, is believed to relieve sleep disturbances [8-10]. In clinical studies, most results are evaluated by interviewing acupuncture subjects [8-10], and there is little objective evidence to reveal the effects of acupuncture. Here, an actigraph, an objective indicator evaluating the quantity and quality of sleep, was applied to an animal model. Placebo-controlled trial models do not work well for most nonpharmacological 
treatments, including acupuncture, because they tend to lack credible placebo controls [11-13]. Subjects receiving acupuncture might have information about its effects, leading to subjective effects of the procedure in humans. On the other hand, animals do not have information about the procedure; therefore, animal models are beneficial for research into acupuncture for insomnia. Some studies on rodents have been reported; however, their extrapolation to humans is restricted due to the difference in sleep patterns between rodents and humans [14].

As large mammals, monkeys have been used for sleep analysis, showing a similar sleep pattern to humans and higher diurnal activity than rodents [15-17]; however, it may be labor-intensive to evaluate the effects of acupuncture on monkeys because of the handling difficulty. On the other hand, pigs as domestic animals can be treated easily by acupuncture. Moreover, there is some information on the relationships between acupoints and complaints [1820]; however, adult pigs have not been fully examined under sleep conditions [21, 22]. Micro-minipigs (MMPigs), a type of miniature pig, are a new porcine model showing atherosclerosis with a high-fat diet $[23,24]$. In order to evaluate the effects of acupuncture on sleep conditions, MMPigs should be examined under sleep conditions.

Here, we examined whether MMPigs show a similar sleep pattern to humans. Moreover, the effects of acupuncture on sleep were evaluated objectively by an actigraph and urinary catecholamine [25-29]. We have tried to establish an animal model to evaluate objectively the effects of alternative medicines on sleep conditions.

\section{Methods}

2.1. Animals. MMPigs were obtained from the breeder (Fuji Micra Inc., Shizuoka, Japan) and kept in a humidity- and temperature-controlled $\left(50 \pm 10 \%, 22 \pm 2^{\circ} \mathrm{C}\right)$ facility with a $12 \mathrm{~h}$ light/dark cycle $(0700 \mathrm{~h}$, lights-on) of Shin Nippon Biomedical Laboratories (Kagoshima, Japan) certified by the Association for the Assessment and Accreditation of Laboratory Animal Care. Light intensity during the day was around $400 \mathrm{~lx}$. Food (ET; Nosan Co., Yokohama, Japan) was supplied to the pigs at around $1600 \mathrm{~h}$. The pigs had free access to water. This study was approved by the Ethics Committee for Animal Experimentation at the Kagoshima University.

2.2. Acupuncture. Subjects assigned to acupuncture were punctured at Dafengmen and pig-Baihui (p-Baihui) according to traditional Chinese veterinary medicine (Figure 1) [18-20]. Dafengmen is located at the midpoint of a line connecting both ears viewed from overhead, which is anatomically similar to the acupoint human-Baihui (hBaihui, Governor Vessel 20: GV20). Baihui is designated as the vertex on the midline based on traditional Chinese medicine [18-20]; therefore, p-Baihui is located at the top of the back near the hip, as shown in Figure 1 [18]. The syringes (diameter $\times$ length: $0.2 \times 50 \mathrm{~mm}$, J type) were obtained from Seirin Co. (Shizuoka, Japan). The acupuncture procedures

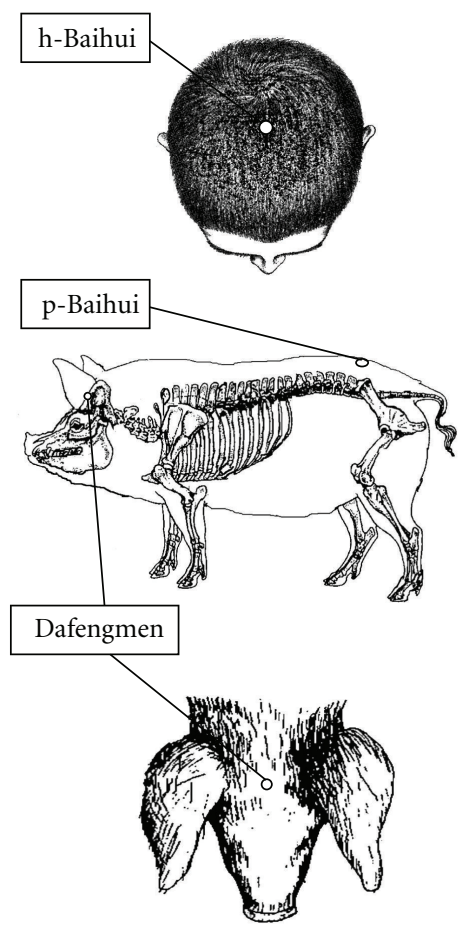

Figure 1: Acupoints for pigs used in the present study are shown in comparison with human acupoints. Human-Baihui (hBaihui, GV20), the midpoint of a line connecting both ears, is an anatomically similar point to Dafengmen in pigs. Pig-Baihui (pBaihui) is on top of the body, which designates the point of Baihui according to traditional Chinese medicine.

were as follows: shallow method, the insertion depth was within $5 \mathrm{~mm}$ for several seconds; deep method, $10 \mathrm{~mm}$ at Dafengmen and $20 \mathrm{~mm}$ at p-Baihui for $20 \mathrm{~min}$. The acupuncture procedures were performed at around 1730$1800 \mathrm{~h}$. Two experiments were performed, as shown in Figure 2. For the first experiment, 6 male pigs (MMPigs 16), one year old, ranging from 8.0 to $10.0 \mathrm{~kg}$ body weight, were used. During days 6-13, MMPigs were treated at pBaihui with the deep or shallow procedure. During days 20 27, MMPigs were treated at Dafengmen with the deep or shallow procedure. For the second experiment to evaluate urinary catecholamine, 4 male pigs (MMPigs 7-10), aged 6 months, ranging from 6.0 to $8.0 \mathrm{~kg}$ body weight, were used. During days 8-11 and days 15-18, MMPigs were treated at p-Baihui or Dafengmen with the deep procedure.

2.3. Actigraph. An actigraph (Octagonal Basic Motionlogger; Ambulatory Monitoring, Inc., Ardsley, NY, USA) was worn $24 \mathrm{~h} / \mathrm{d}$ on the body of the pigs for 2-3 consecutive weeks (Figure 2) $[25,26]$. We used Zero-Crossing Mode for quantification of movement with ACTION-W 2.0 software. As parameters for sleep conditions, total sleep time (TST), sleep efficiency (SE), and actual activity counts were according to an algorithm based on the human algorithm. We analyzed three or four consecutive days' data for the respective treatment $[30,31]$. 


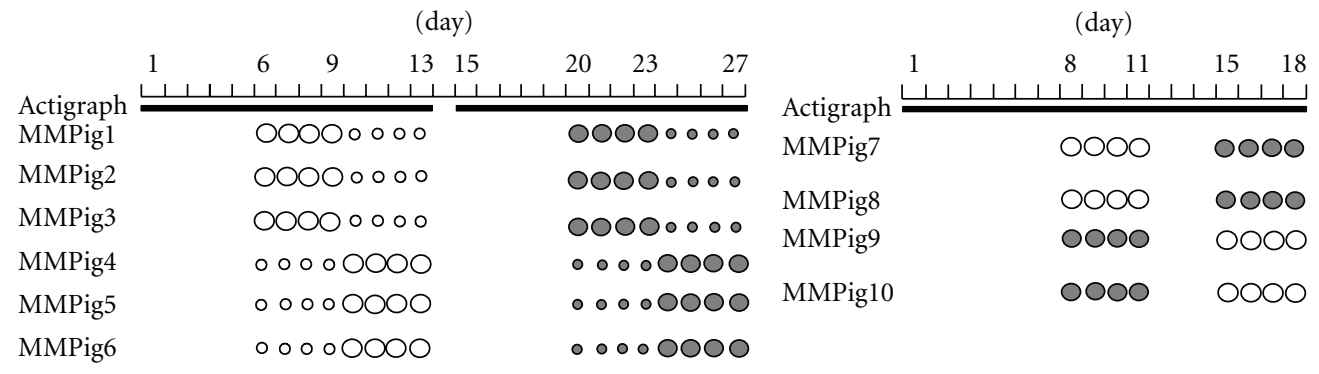

(a) Experiment 1

(b) Experiment 2

Figure 2: The schedule of acupuncture for experiments 1 and 2 is shown. Solid line means the time when the pig was equipped with the actigraph. Open and closed circles mean the acupuncture procedure performed at p-Baihui and Dafengmen (see Section 2), respectively. Small and large circles mean shallow and deep procedures (see Section 2), respectively.

2.4. Urinary Analysis. In the metabolic cages, MMPigs were housed on metal slats, so that urine could be collected in containers. Urine was collected from 1900 to $0900 \mathrm{~h}$ on days 2-4, days 8-11, and days $15-18$ in experiment 2 (Figure 2). The urine was centrifuged, and then $1 \mathrm{~mL}$ of $0.1 \mathrm{~mol} / \mathrm{L}$ EDTA was added to $10 \mathrm{~mL}$ urine [27-29]. Thereafter, the supernatant was frozen and stored at $-20^{\circ} \mathrm{C}$. Norepinephrine and epinephrine were determined with solid-phase extraction and HPLC with electrochemical detection. The values are expressed as the means of the amount excreted (concentration $(\mathrm{mg} / \mathrm{mL}) \times$ urine volume for $14 \mathrm{~h}(\mathrm{~mL}))$ of the urine collected.

2.5. Data Analysis. Values are shown as the respective data. Statistical analysis was performed using the paired Student's $t$-test. $P<0.05$ was considered significant.

\section{Results}

3.1. Actigraph. Sleep conditions of MMPigs were evaluated by the actigraph. As representative data for humans (48 years old, male) and MMPigs (MMPig 1), they were inactive for some hours in the nocturnal phase (Figure 3). The human and MMPig data showed 89.7 and $85.3 \%$ in SE; 323 and $307 \mathrm{~min}$ in TST; 7040 and 8996 in activity, respectively. As a result, MMPigs showed continuous high activity counts in the diurnal phase, indicating that MMPigs are diurnal animals. Sleep onset seems to correspond to lights off, since they were suddenly inactive at around 1900 h. MMPigs did not show any significant differences in activity counts for 20 min during treatment with the deep procedure at $\mathrm{p}$-Baihui or Dafengmen, as compared with untreated MMPigs (pBaihui, $6781 \pm$ 286; Dafengmen, $6413 \pm 756$; untreated, $6850 \pm 565)$. In clinical cases, acupuncture improves sleep conditions at night when the procedure is applied; therefore, we observed the effects of acupuncture on sleep conditions at night $(2200-0400 \mathrm{~h})$. As shown in Figure 3, slept at around 1900 and waking at around 0600 showed higher activity; therefore, we analyzed data during 2200-0400, corresponding to the middle of the lights-off period to evaluate sleep quality. As shown in Figure 4 as the results of experiment 1, MMPigs treated with a shallow or deep procedure at $\mathrm{p}$-Baihui showed no significant differences in
TABLE 1: Effect of acupuncture at different acupoints on sleep conditions and urinary catecholamine.

\begin{tabular}{lccc}
\hline & Untreated & p-Baihui & Dafengmen \\
\hline SE $(\%)$ & $31.8 \pm 6.0$ & $37.8 \pm 8.5$ & $51.9 \pm 7.3$ \\
TST $(\min )$ & $114 \pm 22$ & $136 \pm 31$ & $187 \pm 27^{* *}$ \\
Activity $\left(10^{3} \times\right.$ counts $)$ & $27.6 \pm 1.3$ & $27.7 \pm 5.4$ & $22.1 \pm 3.7$ \\
Norepinephrine $(\mathrm{ng})$ & $870 \pm 124$ & $693 \pm 352$ & $467 \pm 18^{*}$ \\
Epinephrine $(\mathrm{ng})$ & $114 \pm 36$ & $168 \pm 89$ & $149 \pm 36$ \\
\hline
\end{tabular}

Data are presented as the means \pm SEM. SE, sleep efficiency; TST, total sleep time. ${ }^{*} P<0.05 ;{ }^{*} P<0.01$ versus untreated MMPigs.

sleep conditions, including SE $(46.1 \pm 9.7 ; 43.7 \pm 10.6 \%)$, TST $(166.0 \pm 35.0 ; 154.3 \pm 37.9 \mathrm{~min})$, and activity counts $(29791 \pm 6449 ; 28937 \pm 7197)$, during $2200-0400 \mathrm{~h}$ from those of untreated MMPigs (SE, $48.7 \pm 7.5 \%$; TST, $175.4 \pm$ $27.0 \mathrm{~min}$; Activity counts, $24207 \pm 4193)$. On the other hand, MMPigs treated with the deep procedure at Dafengmen showed significantly higher SE $(63.6 \pm 7.1 \%)$ and TST $(229.0$ $\pm 25.7 \mathrm{~min})$ and lower activity counts $(16895 \pm 2872)$ during 2200-0400 $\mathrm{h}$ than those of untreated MMPigs; however, MMPigs treated with the shallow procedure at Dafengmen showed no significant differences in sleep-related parameters during 2200-0400 h, including SE $(56.1 \pm 6.0 \%)$, TST (202.1 $\pm 21.7 \mathrm{~min})$, and activity counts $(20543 \pm 2770)$, compared with those of untreated MMPigs.

3.2. Urinary Catecholamine. In experiment 2, MMPigs treated with deep acupuncture at Dafengmen showed significantly higher TST during 2200-0400 h than untreated MMPigs (Table 1). MMPigs used in experiment 2 showed relatively lower SE, lower TST, and higher activity counts than MMPigs in experiment 1 before acupuncture treatment, probably due to the age difference. Under these conditions, MMPigs treated with the deep procedure at Dafengmen showed significantly lower values in norepinephrine in urine collected from 1900 to $0900 \mathrm{~h}$ than untreated MMPigs. On the other hand, MMPigs treated with the deep procedure at p-Baihui showed similar values in urinary norepinephrine to untreated MMPigs. There were no significant differences in urinary epinephrine among the three groups (Table 1). 


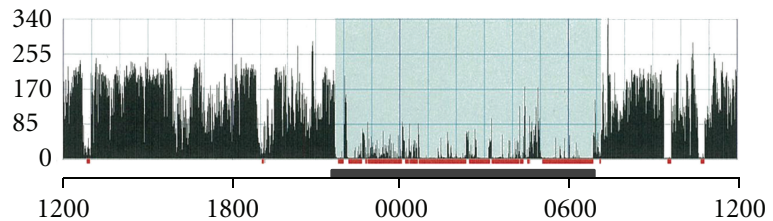

(a)

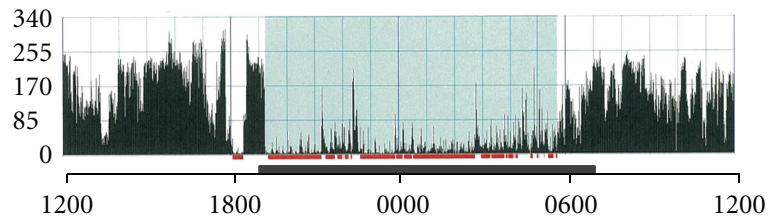

(b)

FIGURE 3: Representative data of actigraph for one day in humans (a) and MMPigs (b). Longitudinal axis means activity counts per minute. Solid horizontal box means the duration of lights off. According to the algorithm for human data analysis, the blue region means lying on the side, and the red mark means sleeping.

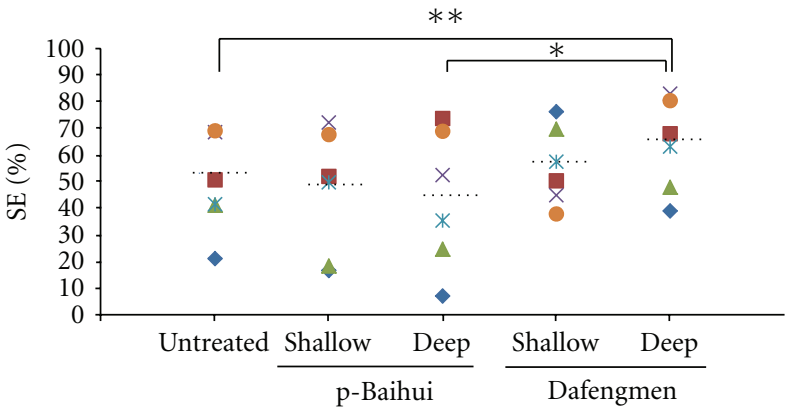

(a)

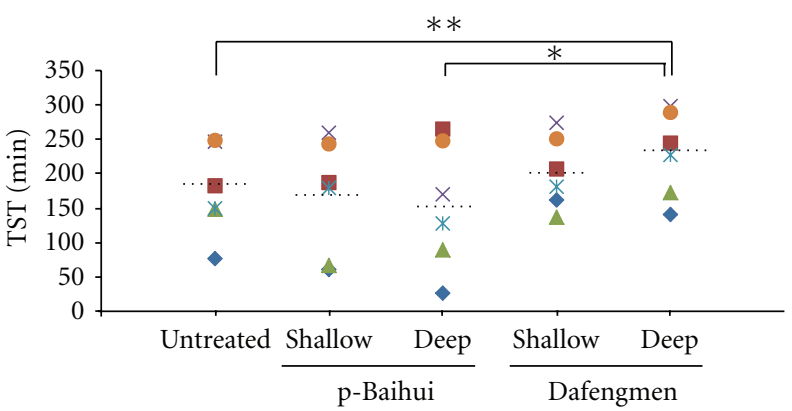

(b)

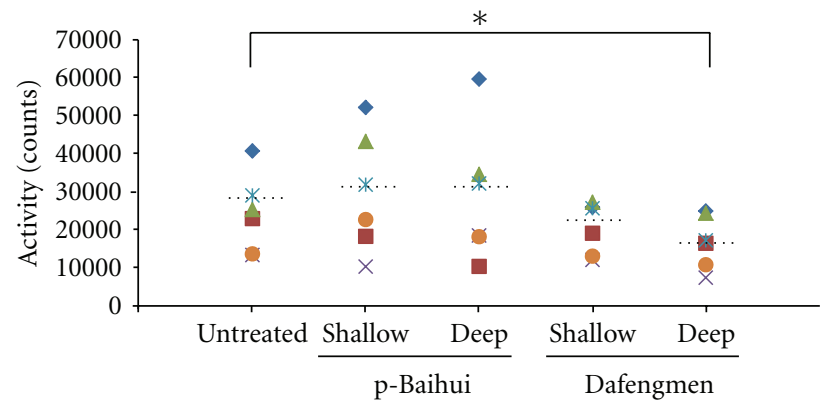

(c)

FIGURE 4: Effects of acupuncture on sleep conditions including sleep efficiency (SE, (a)), total sleep time (TST, (b)), and activity (c) during 2200-0400 h when acupuncture was performed. Different color marks denote the respective MMPigs. Horizontal dotted lines denote the average of the respective data. ${ }^{*} P<0.05,{ }^{* *} P<0.01$, between indicated pairs by the paired Student's $t$-test.

\section{Discussion}

In the present study, we used the smallest pig available for animal experiments to evaluate the effects of acupuncture on sleep conditions using an actigraph and urinary catecholamine. Acupuncture has beneficial effects on sleep conditions only when treated with the deep procedure at an acupoint (Dafengmen) that is anatomically similar point to humans (Baihui, GV20). To the best of our knowledge, this is the first report describing the objectively beneficial effects of acupuncture on sleep conditions using large mammals.

Acupuncture has been widely applied clinically to people with sleep disturbances [7-10]; however, there are few objective data. One of the purposes of the present study was to establish an animal model to evaluate sleep conditions in order to obtain objective data on acupuncture procedures. For analysis of the effects of acupuncture on digestive function, rats and dogs have been used as an animal model
[32-34]; however, rats are not diurnal animals and have different sleep conditions from humans. On the other hand, the MMPigs used in the present study are typical diurnal animals (Figure 3). Namely, MMPigs were inactive for a long period in the nocturnal phase and continuously active in the daytime. There is a long history of pigs coexisting with humans [18-20]; therefore, humans and pigs might have similar living patterns. Moreover, MMPigs, which have been developed recently in Japan, are small and placid, which are beneficial characteristics for an animal model. MMPigs adapted easily to the harness equipped with an actigraph, a device for evaluating sleep conditions. To obtain objective data on the effects of acupuncture, we used the actigraph for several successive days. As shown in Figure 4, untreated MMPigs showed 20-70\% of SE, indicating that the living environment of MMPigs may be somewhat stressful. Namely, MMPigs are models of sleep disturbance. In addition, we measured urinary catecholamine during the 
nocturnal phase, which is an appropriate biomarker for sleep conditions [27-29]. Here, we used MMPigs, diurnal animals, equipped with an actigraph to evaluate the effects of acupuncture on sleep conditions.

The physiological mechanisms of acupuncture have been controversial, and hence the method of needling practices, optimal mode of stimulation, and selection of acupuncture points are complicated. In the clinical procedure for patients with sleep disturbances, Baihui is used as the acupuncture point $[9,10]$. According to traditional Chinese medicine, Baihui is located at the vertex on the midline, so h-Baihui and p-Baihui are located at different points anatomically (Figure 1) [18-20]; therefore, we selected another acupoint, Dafengmen, which is anatomically similar to h-Baihui. As a result, only acupuncture at Dafengmen for a long duration improved SE, TST, and total activity during the night, and suppressed urinary excretion of norepinephrine. The duration of stimulation may be related to the beneficial effects. Moreover, these results mean that the acupoint for improvement of sleep quality is anatomically similar to h-Baihui (GV20) rather than p-Baihui determined by traditional Chinese medicine. In dogs, acupuncture at GV20 (located on the head) induced sedative effects based on EEG analysis, indicating that stimulation of GV20 by acupuncture may affect the central nervous system related to the hypothalamus [35-37]. On the other hand, stimulation of GV20 by acupuncture in rats increased locomotor activity [38], indicating that the effects of acupuncture at GV20 may be different outcomes in species. GV20 may be located in an area governed by the trigeminal nerve and/or greater occipital nerve in different species $[35,36]$. Acupuncture stimulation may be transferred to hypothalamic parasympathetic nerve systems involved in sleep or wake conditions through trigeminal and/or greater occipital nerves because there is a crosstalk between nerve systems $[39,40]$. Regarding the relationship between neurological anatomy and the physiological response against acupuncture, further experiments using MMPigs will be required.

In conclusion, we have established an animal model to evaluate the effects of acupuncture on sleep conditions. Beneficial effects occur when acupuncture is performed at a specific point, indicating that the effects may be induced by transferring the stimulus to the central nervous system, including the sleep center, through trigeminal and/or greater occipital nerves.

\section{Conflict of Interests}

The authors have indicated no financial conflicts of interest.

\section{Acknowledgments}

The authors thank Chiko Yumiba for administrative assistance and Daniel Mrozek for editing the paper. They also greatly thank Nobuo Kanzaki for encouraging them to continue with this work. This work was supported by grants from the Ministry of Education, Culture, Sports, Science and Technology, Japan, and from the Kodama Memorial
Fund for Medical Research. K.-I. Takeishi and M. Horiuchi contributed equally to the present study. T. Takeuchi died during the preparation of the paper.

\section{References}

[1] K. Spiegel, R. Leproult, and E. Van Cauter, "Impact of sleep debt on metabolic and endocrine function," The Lancet, vol. 354, no. 9188, pp. 1435-1439, 1999.

[2] M. Nagai, S. Hoshide, and K. Kario, "Sleep duration as a risk factor for cardiovascular disease-a review of the recent literature," Current Cardiology Reviews, vol. 6, no. 1, pp. 5461, 2010.

[3] M. Kopasz, B. Loessl, M. Hornyak et al., "Sleep and memory in healthy children and adolescents - a critical review," Sleep Medicine Reviews, vol. 14, no. 3, pp. 167-177, 2010.

[4] J. M. Gregory, "Sleep: a good investment in health and safety," Journal of Agromedicine, vol. 13, no. 2, pp. 119-131, 2008.

[5] J. D. Kranzler and R. M. Gendreau, "Role and rationale for the use of milnacipran in the management of fibromyalgia," Neuropsychiatric Disease and Treatment, vol. 6, no. 1, pp. 197208, 2010.

[6] V. Srinivasan, J. Singh, S. R. Pandi-Perumal, G. M. Brown, D. W. Spence, and D. P. Cardinali, "Jet lag, circadian rhythm sleep disturbances, and depression: the role of melatonin and its analogs," Advances in Therapy, vol. 27, no. 11, pp. 796-813, 2010.

[7] J. Sarris and G. Byrne, "A systematic review of insomnia and complementary medicine," Sleep Medicine Reviews, vol. 15, no. 2, pp. 99-106, 2011.

[8] K. D. Phillips and W. D. Skelton, "Effects of individualized acupuncture on sleep quality in HIV disease," The Journal of the Association of Nurses in AIDS Care, vol. 12, no. 1, pp. 2739, 2001.

[9] W. F. Yeung, K. F. Chung, S. P. Zhang, T. G. Yap, and A. C. K. Law, "Electroacupuncture for primary insomnia: a randomized controlled trial," Sleep, vol. 32 , no. 8, pp. 10391047, 2009.

[10] W. F. Yeung, K. F. Chung, K.C. Tso, S. P. Zang, Z. J. Zang, and L. M. Ho, "Electoroacupuncture for residual insomnia associated with major depressive disorder: a randomized controlled trial," Sleep, vol. 34, no. 6, pp. 807-815, 2011.

[11] M. Haake, H.-H. Muller, C. Schade-Brittinger et al., "German Acupuncture Trials (GERAC) for chronic low back pain: randomized, multicenter, blinded, parallel-group trial with 3 groups," Archives of Internal Medicine, vol. 167, no. 17, pp. 1892-1898, 2007.

[12] D. C. Cherkin, K. J. Sherman, A. L. Avins et al., "A randomized trial comparing acupuncture, simulated acupuncture, and usual care for chronic low back pain," Archives of Internal Medicine, vol. 169, no. 9, pp. 858-866, 2009.

[13] P. White, G. Lewith, V. Hopwood, and P. Prescott, "The placebo needle, is it a valid and convincing placebo for use in acupuncture trials? A randomised, single-blind, cross-over pilot trial," Pain, vol. 106, no. 3, pp. 401-409, 2003.

[14] A. A. Borbely, "Effects of light on sleep and activity rhythms," Progress in Neurobiology, vol. 10, no. 1, pp. 1-31, 1978.

[15] I. V. Zhdanova, D. A. Geiger, A. L. Schwagerl et al., "Melatonin promotes sleep in three species of diurnal nonhuman primates," Physiology \& Behavior, vol. 75, no. 4, pp. 523-529, 2002.

[16] K. C. Hsieh, E. L. Robinson, and C. A. Fuller, "Sleep architecture in unrestrained rhesus monkeys (Macaca mulatta) 
synchronized to 24-hour light-dark cycles," Sleep, vol. 31, no. 9, pp. 1239-1250, 2008.

[17] C. E. Barrett, P. Noble, E. Hanson, D. S. Pine, J. T. Winslow, and E. E. Nelson, "Early adverse rearing experiences alter sleep-wake patterns and plasma cortisol levels in juvenile rhesus monkeys," Psychoneuroendocrinology, vol. 34, no. 7, pp. 1029-1040, 2009.

[18] C. Li and B. Hang, Eds., Chugoku Jyui Shinkyu Zufu, Daiichi Shuppan, 1998.

[19] W. W. Chan, K. Y. Chen, H. Liu, L. S. Wu, and J. H. Lin, "Acupuncture for general veterinary practice," Journal of Veterinary Medical Science, vol. 63, no. 10, pp. 1057-1062, 2001.

[20] G. Habacher, M. H. Pittler, and E. Ernst, "Effectiveness of acupuncture in veterinary medicine: systematic review," Journal of Veterinary Internal Medicine, vol. 20, no. 3, pp. 480488, 2006.

[21] A. Forslid, B. Andersson, and S. Johansson, "Observations on normal EEG activity in different brain regions of the unrestrained swine," Acta Physiologica Scandinavica, vol. 128, no. 3, pp. 389-396, 1986.

[22] S. Robert and A. Dallaire, "Polygraphic analysis of the sleepwake states and the REM sleep periodicity in domesticated pigs (Sus scrofa)," Physiology \& Behavior, vol. 37, no. 2, pp. 289-293, 1986.

[23] N. Miyoshi, M. Horiuchi, Y. Inokuchi et al., "Novel microminipig model of atherosclerosis by high fat and high cholesterol diet, established in Japan," In Vivo, vol. 24, no. 5, pp. 671-680, 2010.

[24] N. Kaneko, K. Itoh, A. Sugiyama, and Y. Izumi, "Microminipig, a non-rodent experimental animal optimized for life science research: preface," Journal of Pharmacological Sciences, vol. 115, no. 2, pp. 112-114, 2011.

[25] S. Q. Zhang, S. Kozuma, M. Tanaka et al., "Studies on fetal forelimb movements by using a wrist actigraph in sheep," Psychiatry and Clinical Neurosciences, vol. 56, no. 3, pp. 283284, 2002.

[26] C. Giannetto, S. Casella, G. Caola, and G. Piccione, "Photic and non-photic entrainment on daily rhythm of locomotor activity in goats," Animal Science Journal, vol. 81, no. 1, pp. 122-128, 2010.

[27] S. J. Koopmans, M. Ruis, R. Dekker, and M. Korte, "Surplus dietary tryptophan inhibits stress hormone kinetics and induces insulin resistance in pigs," Physiology \& Behavior, vol. 98, no. 4, pp. 402-410, 2009.

[28] E. Peeters, A. Neyt, F. Beckers, S. De Smet, A. E. Aubert, and R. Geers, "Influence of supplemental magnesium, tryptophan, vitamin $\mathrm{C}$, and vitamin $\mathrm{E}$ on stress responses of pigs to vibration," Journal of Animal Science, vol. 83, no. 7, pp. 15681580, 2005.

[29] J. Zhang, R. C. W. Ma, A. P. S. Kong et al., "Relationship of sleep quality and quality with 24-hour urinary catecholamines and salivary awakening cortisol in healthy middleaged adults," Sleep, vol. 34, no. 2, pp. 225-233, 2011.

[30] P. S. Hinds, M. J. Hockenberry, J. S. Gattuso et al., "Dexamethasone alters sleep and fatigue in pediatric patients with acute lymphoblastic leukemia," Cancer, vol. 110, no. 10, pp. 2321-2330, 2007.

[31] K. H. Zeiders, L. D. Doane, and E. K. Adam, "Reciprocal relations between objectively measured sleep patterns and diurnal cortisol rhythms in late adolescence," Journal of Adolescent Health, vol. 48, no. 6, pp. 566-571, 2011.

[32] K. Murase and K. Kawakita, "Diffuse noxious inhibitory controls in anti-nociception produced by acupuncture and moxibustion on trigeminal caudalis neurons in rats," Japanese Journal of Physiology, vol. 50, no. 1, pp. 133-140, 2000.

[33] H. Tada, M. Fujita, M. Harris et al., "Neural mechanism of acupuncture-induced gastric relaxations in rats," Digestive Diseases and Sciences, vol. 48, no. 1, pp. 59-68, 2003.

[34] M. Iwa, C. Strickland, Y. Nakade, T. N. Pappas, and T. Takahashi, "Electroacupuncture reduces rectal distensioninduced blood pressure changes in conscious dogs," Digestive Diseases and Sciences, vol. 50, no. 7, pp. 1264-1270, 2005.

[35] W. E. Shankland II, "The trigeminal nerve. Part IV: the mandibular division," Journal of Craniomandibular Practice, vol. 19, no. 3, pp. 153-161, 2001.

[36] M. S. Kim and T. C. Nam, "Electroencephalography (EEG) spectral edge frequency for assessing the sedative effect of acupuncture in dogs," Journal of Veterinary Medical Science, vol. 68, no. 4, pp. 409-411, 2006.

[37] M. S. Kim, K. S. Soh, T. C. Nam, K. M. Seo, and G. Litscher, "Evaluation of sedation on electroencephalographic spectral edge frequency 95 in dogs sedated by acupuncture at GV20 or Yintang and sedative combination," Acupuncture and ElectroTherapeutics Research International Journal, vol. 31, no. 3-4, pp. 201-212, 2006.

[38] Y. H. Chang, M. T. Hsieh, and J. T. Cheng, "Increase of locomotor activity by acupuncture on Bai-Hui point in rats," Neuroscience Letters, vol. 211, no. 2, pp. 121-124, 1996.

[39] H. Izumi and K. Karita, "Vasodilator responses following intracranial stimulation of the trigeminal, facial and glossopharyngeal nerves in the cat gingiva," Brain Research, vol. 560, no. 1-2, pp. 71-75, 1991.

[40] N. Salih, P. I. Baumler, M. Simang, and D. Irnich, "Deqi Sensations without cutaneous sensory input: results of an RCT," BMC Complementary and Alternative Medicine, vol. 10, article 81, 2010. 


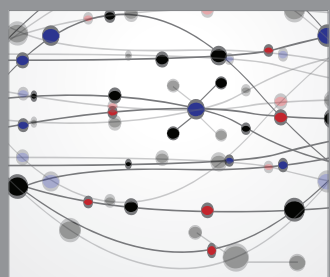

The Scientific World Journal
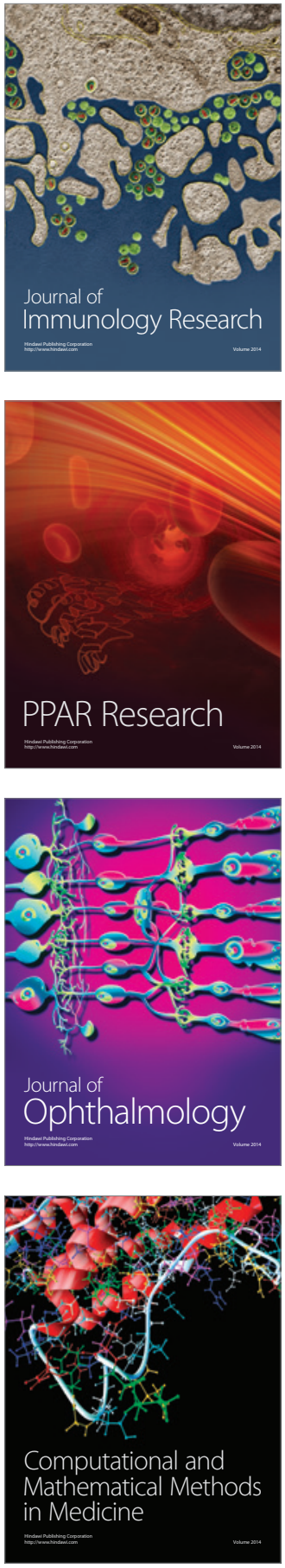

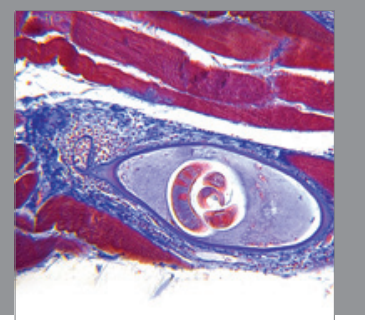

Gastroenterology

Research and Practice
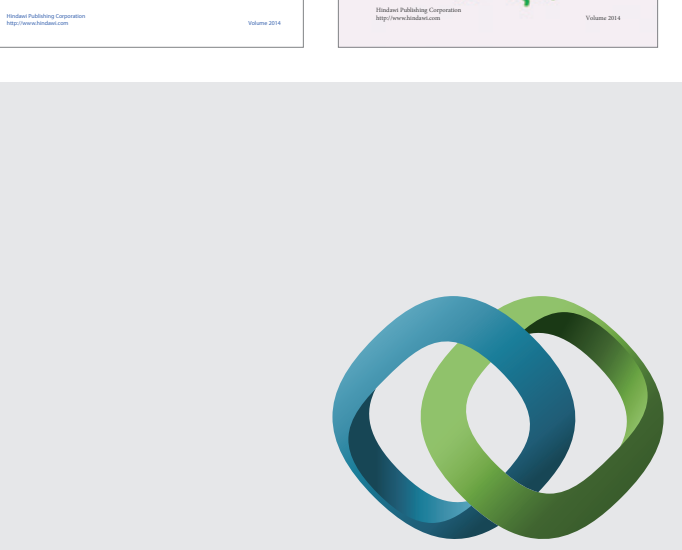

\section{Hindawi}

Submit your manuscripts at

http://www.hindawi.com
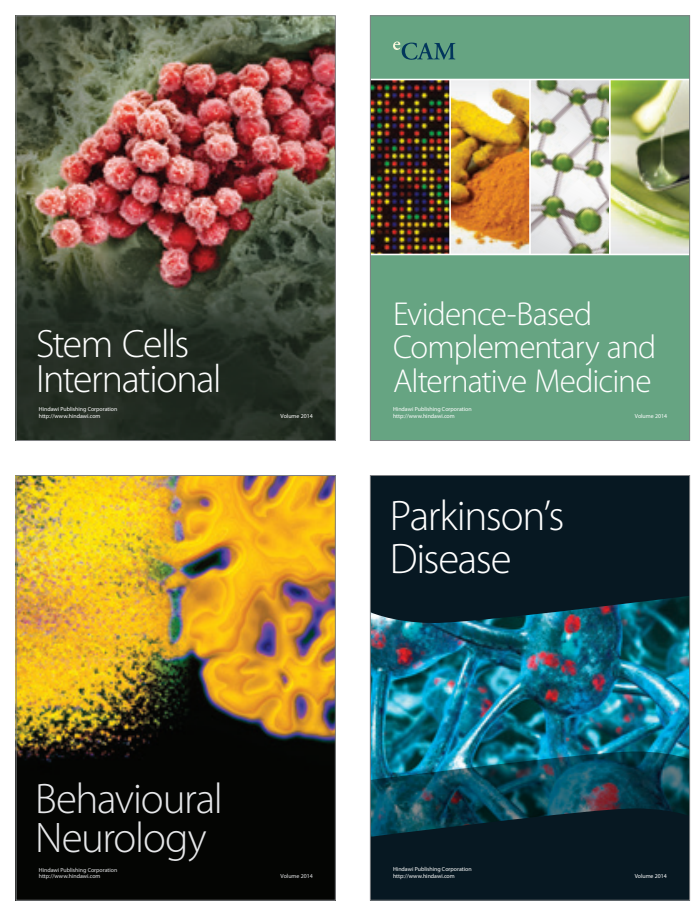

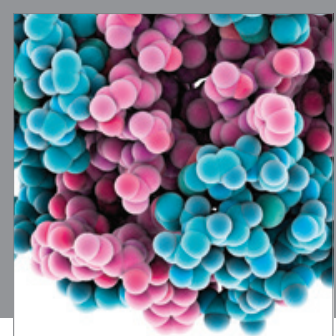

Journal of
Diabetes Research

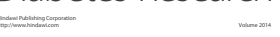

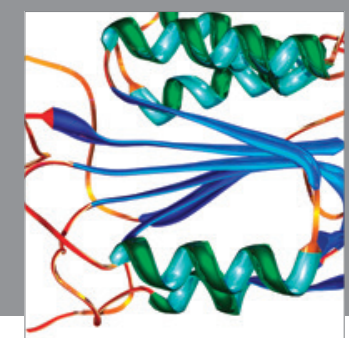

Disease Markers
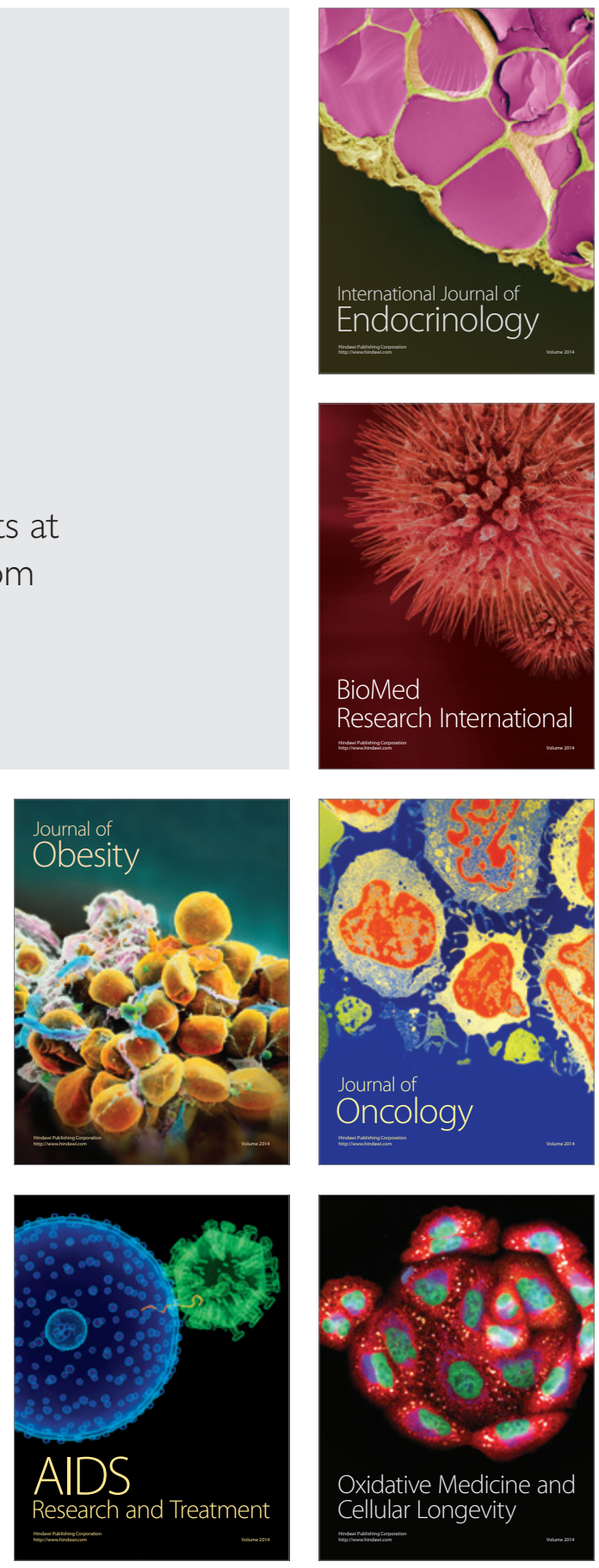